\title{
Analysis and Simulation about a Simplified Model for Modulation and Demodulation of Ship-borne Single Channel Monopulse Radar
}

\author{
Xudong Zhang, Haiyu Ji \\ China Satellite Maritime Tracking \& Control Department, 214431 Jiangyin Jiangsu, China
}

\begin{abstract}
A simplified mathematical model for modulation and demodulation of a single channel monopulse (SCM) system is proposed, which is based on a ship-borne pulse radar S-band guided receiver. Using the proposed mathematical model, the modulation and demodulation of single-channel and multi-channel signals were simulated respectively, and the factors influencing the modulation and demodulation of the SCM system were analysed.
\end{abstract}

\section{Introduction}

For the purpose of simplifying channels, a ship-borne monopulse radar guidance receiver adopts the SCM system. Therefore, it is necessary to synthesize the sum, azimuth and pitching signals to one channel in the guided feed [1]. The synthetic principle is shown in Figure 1.

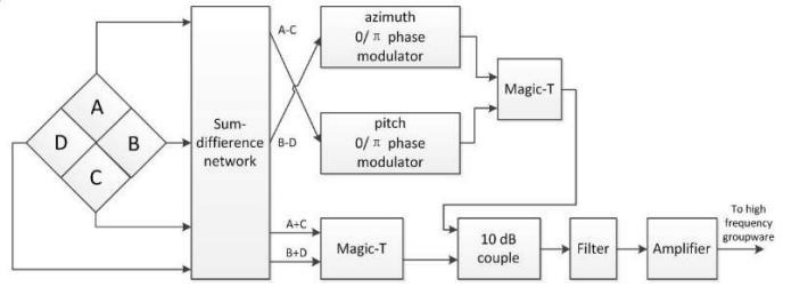

Figure 1. Block diagrams of synthetic principle.

When the four guide antennas receive signals, modulation is performed in the guidance central body. Firstly, the sum, azimuth and pitching signals are generated through the sum-difference network and Magic-T. Then square wave is used to perform $0 / \pi$ modulation on the azimuth and pitching signals. After the Magic-T, a 10dB coupler is added to synthesize the three signals eventually. After amplification, mixing, filtering and quadrature sampling, the angle error voltage is finally demodulated in the DSP [2-4].

\section{A proposal of the simplified model}

\subsection{Modulation and demodulation of one signal}

A signal $y=f_{0}(t)$ is modulated with square-wave as shown in equation (1):

$$
T z(t)=\left\{\begin{array}{lr}
1 & n T \leq t \leq n T+\frac{T}{2} \\
-1 & n T+\frac{T}{2} \leq t \leq(n+1) T
\end{array}\right.
$$

Among them, $n=(1,2,3 \ldots)$. Then the modulated signal is $y=f_{0}(t) T z(t)$, which is

$$
y=\left\{\begin{array}{rr}
f_{0}(t) & n T \leq t \leq n T+\frac{T}{2} \\
-f_{0}(t) & n T+\frac{T}{2} \leq t \leq(n+1) T
\end{array}\right.
$$

To recover $y=f_{0}(t)$ from the modulated signal, you can multiply the modulated signal $y=f_{0}(t)$ by $T z(t)$ again.

Then the signal becomes

$$
y=f_{0}(t) T z(t) T z(t)
$$

If this signal is superimposed with a signal $y=f_{l}(t)$ which is not modulated with square-wave, the resulting signal is

$$
y=\left\{\begin{array}{rr}
f_{1}(t)+f_{0}(t) & n T \leq t \leq n T+\frac{T}{2} \\
f_{1}(t)-f_{0}(t) & n T+\frac{T}{2} \leq t \leq(n+1) T
\end{array}\right.
$$

It is difficult to demodulate $y=f_{0}(t)$ from equation (4). But some information can be extracted from some given condition.

If the signals $f_{0}(t), f_{l}(t)$ are limited to a short-term amplitude-stabilized DC signal or periodic signal, and the frequency of the limited periodic signal is much greater than the frequency of the modulated signal, then the average value of the signal can be extracted after every demodulation period [5-6]. Since $f_{l}(t)$ is not modulated, the integral term is zero when multiplied by $T z(t)$, so as to extract the amplitude information of $f_{0}(t)$ [7-8], which is

$$
y=\frac{\int_{0}^{\frac{T}{2}}\left(f_{1}(t)+f_{0}(t)\right) T z(t) d t+\int_{\frac{T}{2}}^{T}\left(f_{1}(t)-f_{0}(t)\right) T z(t) d t}{T}
$$

Substituting equation (1) into equation (5), the signal $y$ is 


$$
y=\frac{\int_{0}^{\frac{T}{2}}\left(f_{1}(t)+f_{0}(t)\right) d t+\int_{\frac{T}{2}}^{T}\left(f_{0}(t)-f_{1}(t)\right) d t}{T}=\frac{\int_{0}^{T} f_{0}(t) d t}{T}
$$

\subsection{Modulation and demodulation of two signals}

If signal $f_{0}(t) T z(t)$ is superimposed with another modulated signal $f_{2}(t) T z^{\prime}(t)$, the resulting signal is

$$
y=f_{0}(t) T z(t)+f_{2}(t) T z^{\prime}(t)
$$

To demodulate $f_{0}(t)$ from the signal represented by equation (7), $T z^{\prime}(t)$ needs to be confined additionally: $T z(t)$ and $T z^{\prime}(t)$ are under the same period and the phase difference is $\pi / 4$ between $T z(t)$ and $T z^{\prime}(t)$.

$$
T z^{\prime}(t)=\left\{\begin{array}{lr}
1 & n T+\frac{T}{4} \leq t \leq n T+\frac{3 T}{4} \\
-1 & n T+\frac{3 T}{4} \leq t \leq(n+1) \frac{5 T}{4}
\end{array}\right.
$$

The signal in equation (7) becomes equation (9).

$$
y=\left\{\begin{array}{cc}
f_{0}(t)-f_{2}(t) \quad n T \leq t \leq n T+\frac{T}{4} \\
f_{0}(t)+f_{2}(t) n T+\frac{T}{4} \leq t \leq n T+\frac{T}{2} \\
f_{2}(t)-f_{0}(t) n T+\frac{T}{2} \leq t \leq n T+\frac{3 T}{4} \\
-f_{0}(t)-f_{2}(t) n T+\frac{3 T}{4} \leq t \leq(n+1) T
\end{array}\right.
$$

Multiply the signal represented by equation (9) with $T z(t)$, equation (10) can be obtained.

$$
y=\left\{\begin{array}{rr}
f_{0}(t)-f_{2}(t) & n T \leq t \leq n T+\frac{T}{4} \\
f_{0}(t)+f_{2}(t) & n T+\frac{T}{4} \leq t \leq n T+\frac{T}{2} \\
f_{0}(t)-f_{2}(t) & n T+\frac{T}{2} \leq t \leq n T+\frac{3 T}{4} \\
f_{0}(t)+f_{2}(t) & n T+\frac{3 T}{4} \leq t \leq(n+1) T
\end{array}\right.
$$

Still taking the averaging method, you can eliminate $f_{2}(t)$ and extract $f_{0}(t)$.

The signal represented by equation (11) can be obtained by multiplying and demodulating the signal represented by equation (9) and $T z^{\prime}(t)$. By taking the averaging method, $f_{0}(t)$ can be eliminated and $f_{2}(t)$ can be extracted.

$$
y=\left\{\begin{array}{cc}
f_{2}(t)-f_{2}(t) & n T \leq t \leq n T+\frac{T}{4} \\
f_{0}(t)+f_{2}(t) & n T+\frac{T}{4} \leq t \leq n T+\frac{T}{2} \\
f_{0}(t)-f_{2}(t) & n T+\frac{T}{2} \leq t \leq n T+\frac{3 T}{4} \\
f_{0}(t)+f_{2}(t) & n T+\frac{3 T}{4} \leq t \leq(n+1) T
\end{array}\right.
$$

\section{Simulation results of modulation and demodulation}

\subsection{Simulation result of one signal}

Three DC signals indicate sum, azimuth and pitching. The sum signal is not modulated, the azimuth signal is modulated by $T z(t)$ and the pitching signal is modulated by $T z^{\prime}(t) . T z(t)$ and $T z^{\prime}(t)$ are square wave signals of period 200, and $T z^{\prime}(t)$ is delayed by 50 relative to $T z(t)$, that is, the phase difference is $\pi / 4$. The algorithm is simulated by a program written by $\mathrm{VC}++$. The left green waveform is the modulated azimuth signal, the left red waveform is the sum signal waveform, the left yellow waveform is the modulated pitching signal. On the right side, the green waveform is the waveform after azimuth demodulation, the red waveform is the waveform after modulation and synthesis, and the yellow waveform is the waveform after pitching demodulation. When the amplitude of the sum and pitching signal is zero and the amplitude of the azimuth signal is 20 , the modulated azimuth signal is shown in the left green waveform of Figure 2, the synthesized signal waveform is shown by the red waveform on the right side of Figure 2.

After demodulation, the azimuth waveform recovers, and the amplitude information can be extracted after averaging. The sum and pitching signal is a square waveform with an average value of 0 .

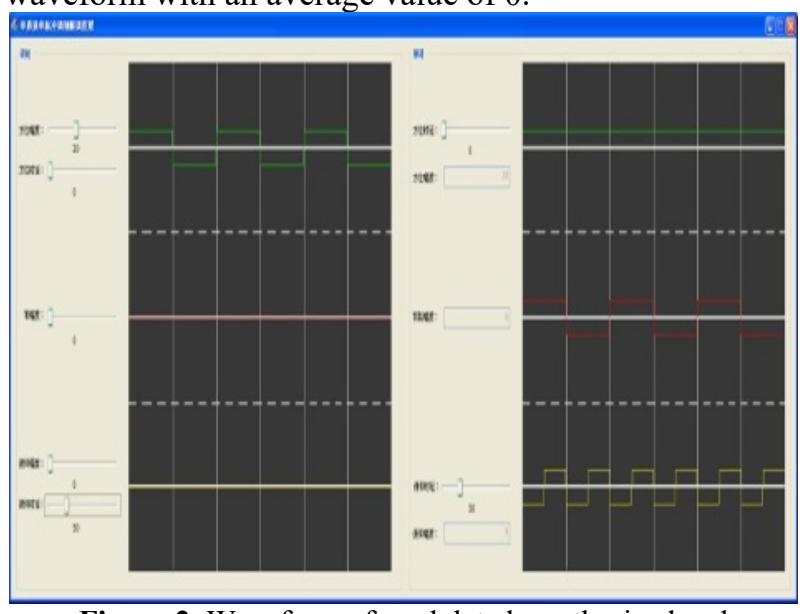

Figure 2. Waveform of modulated, synthesized and demodulated azimuth signal.

\subsection{Simulation result of two signals}

The amplitude of the sum signal is set to 20 , which is superimposed with the azimuth signal. As can be seen from Figure 3, the superimposed waveform has an overall shift of the azimuth signal by 20 , but the mean is the same as the original sum signal amplitude, and the demodulated azimuth signal is also with an offset of 20 , the mean is also consistent with the amplitude of the original azimuth signal, and the pitching signal after demodulation is a staircase signal with an average of 0 . 


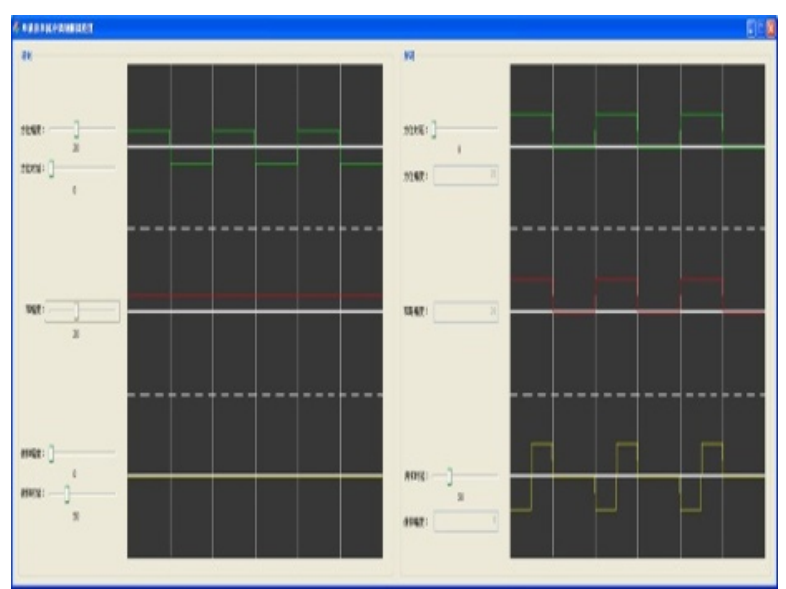

Figure 3. Waveform of modulated, synthesized and demodulated sum and azimuth signals.

\subsection{Simulation result of three signals}

The waveform can be seen in Figure 4 when the amplitude of the pitching signal is added to 10 and superimposed on the sum signal and the azimuth signal. Although they become more complicated, the amplitude of each signal can be extracted after averaging.

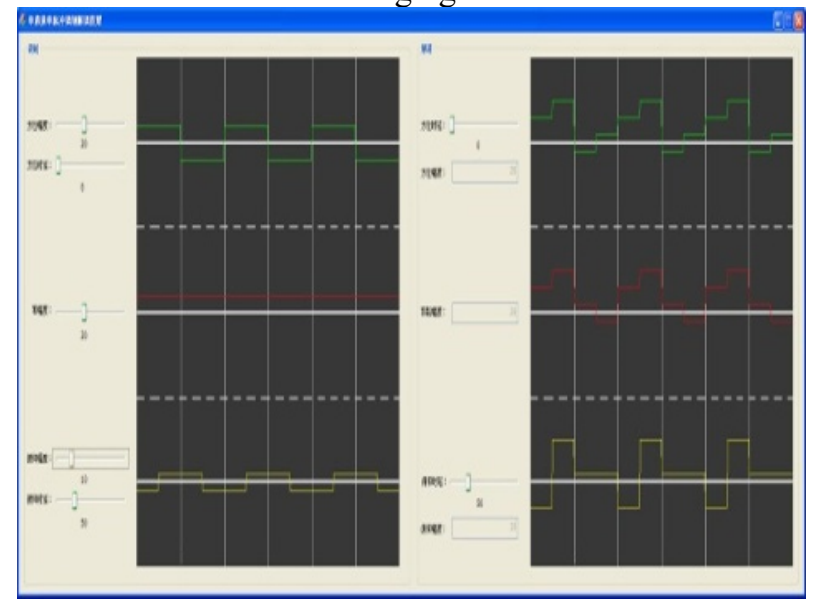

Figure 4. Waveform of modulated, synthesized and demodulated sum, azimuth and pitching signals.

\subsection{Further analysis of simulation results}

The above section describes the modulation and demodulation of signals under ideal conditions. If the time sequence between the two modulation signals and the time sequence between modulation and demodulation signals does not meet the above requirements, the simulation results are given below.

From Figure 5 to Figure 8, it can be seen that regardless of the value of the delay adjustment of the modulated and demodulated signals, the average value of the sum signal after synthesis is always the same, and the amplitude of the sum signal does not affect the average value of azimuth and pitching signals after demodulation. But the time delay value has a direct effect on the demodulated azimuth and pitching average value. If the adjustment is not appropriate, cross coupling will occur.

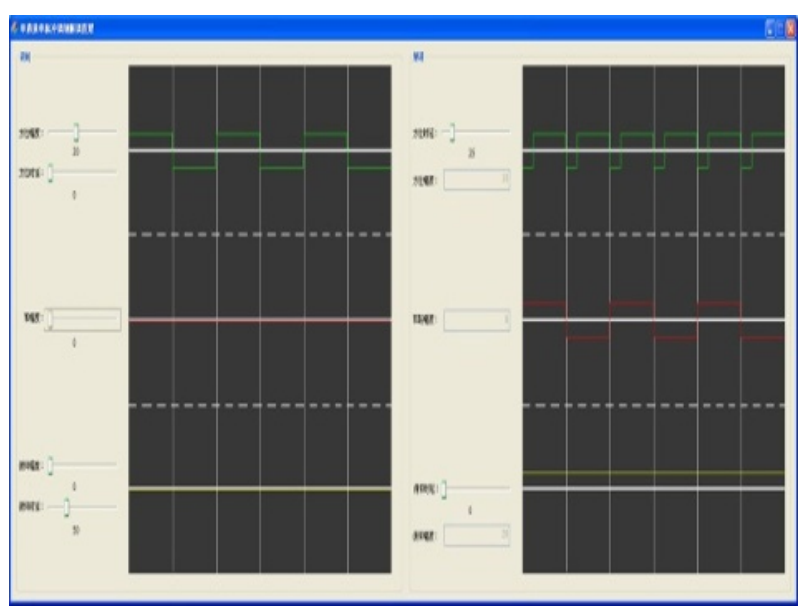

Figure 5. Simulation result of modulated and demodulated azimuth signals when time sequence demands of azimuth signal are not fulfilled.

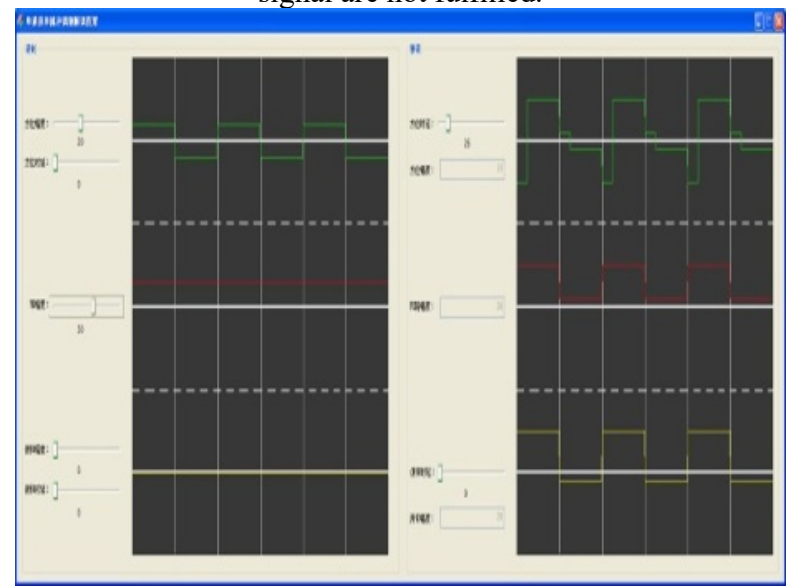

Figure 6. Simulation result of superimposed pitching and sum signals when time sequence demands of azimuth signal are not

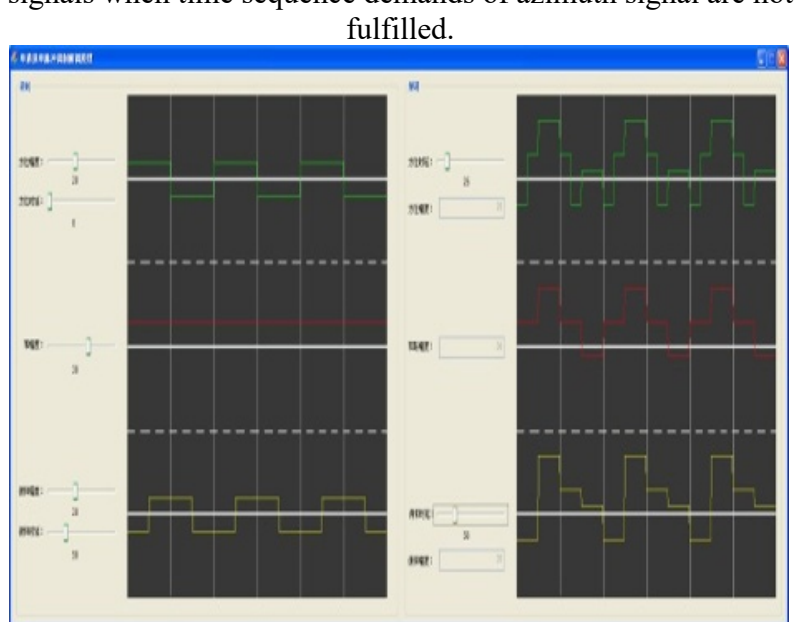

Figure 7. Simulation result of pitching and sum signals when time sequence demands of azimuth signal are not fulfilled. 


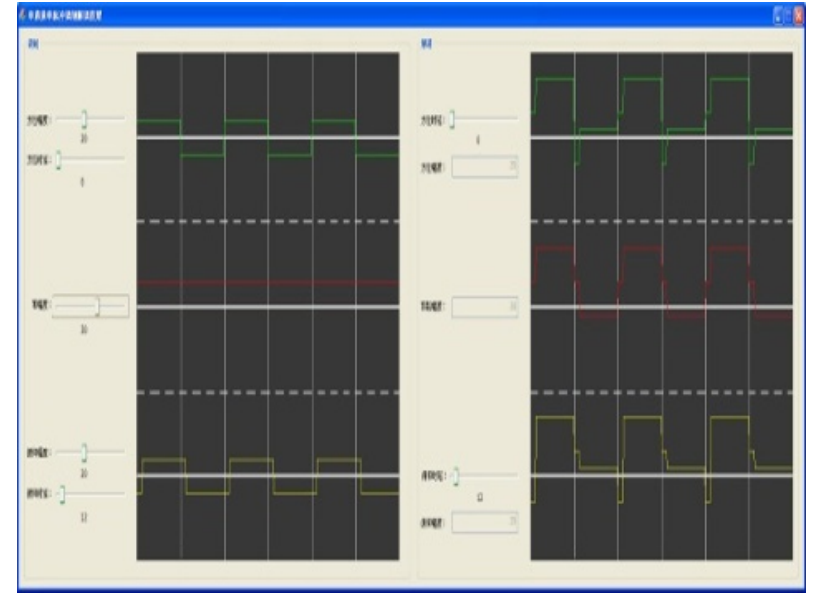

Figure 8. Simulation result of three signals when time sequence demands of azimuth and pitching signal are not fulfilled.

\section{Conclusion}

This paper analyses the principle of modulation and demodulation of a SCM system receiver for ship-boren radar and gives a mathematical implementation method. Using $\mathrm{VC}++$ to write simulation program, the influence of signal amplitude and delay on SCM modulation and demodulation is further analysed, and the reason of crosscoupling in this system is analysed. It can be useful to the fault analysis and troubleshooting of the equipment when using the simulation software to compare the simulation results under different conditions with the actual test waveforms.

\section{References}

1. M. Dietmar, A Technology for Tuture Electronic Warfare Systems, International Conference on Radar System, 13-15, (1999)

2. T. W. Fields, D. L. Sharpin, J. B. Tsui, Digital channelized IFM receiver, IEEE International Microwave Symposium, 77-78, (1994)

3. L. Shaocheng, J. biao, Y. Hujun, S. Tao, A Real-time Algorithm for Signal Detection Based on Autocorrelation at Low SNR, IEEE International Conference on Signal Processing, 129-130, (2010)

4. W. Jinsong, L. Hailin, Analysis on the Guidence System of Shipborne Monopulse Instrumentation Radar, Modern Rader, 05, (2009)

5. X. Xingyuan, G. Quanhui, The Angular Error Demodulation for SCM Tracking System, J. Telemetry Tracking \& Command, 05, (1998)

6. S. Lacheta, P. K. Gupta, J. K. Hota, Generic Digital Monopulse Tracking Receiver for Advanced Communication Satellites, IEEE International Conference on Electronics Computing and Communication Technologies, 1-5, (2015)

7. T. L. Sheret, B Allen, C. G. Parrini, Monopulse Sum and Difference Signals with Compensation for A Failed Feed Element, Let Microwaves Antennas \& Propagation, 10, 645-650, (2016)
8. A. K. Ray, R. C. Shit, R. S. Yadav, Development of C-Band RF Front-end of Precision Coherent Monopluse C-Band Radar, Defence Science Jounal, 64, 358-365, (2014) 